\title{
Single-cell atlases dissect tissue destruction by SARS-CoV-2
}

regenerative
strategies are
initiated to
re-establish
alveolar epi-
thelial cells
lost to infec-
tion, but ...
a serial failure
of epithelial
progenitors
to regenerate
may eventually
lead to lung
failure

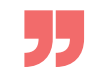

Severe SARS-CoV-2 infection can cause acute respiratory distress syndrome and multi-organ failure. Now, two studies, published back-to-back in Nature, provide new insights into the pathophysiology of severe COVID-19 at single-cell resolution.

Melms et al. carried out singlenucleus RNA sequencing (snRNA-seq) of $\sim 116,000$ nuclei from snap-frozen lungs that were collected shortly after death from 19 individuals who died of COVID-19, as well as 7 control lungs. They found that lungs from COVID-19-decedents were highly inflamed and had dense infiltrations of aberrantly activated macrophages. There was evidence of plasma cells capable of producing neutralizing antibodies, but $\mathrm{T}$ cell responses appeared to be impaired - indicating that, despite potentially sufficient humoral immune responses, inadequate $\mathrm{T}$ cell responses may contribute to lethal COVID-19.

The authors found evidence that SARS-CoV-2 not only directly damages the epithelium but also affects its regeneration. Alveolar type II (AT2) epithelial cells were found to be compromised in their transition to alveolar type I (AT1) cells, with increased numbers of cells arrested at a 'damage-associated transient progenitor' (DATP) cell state, which

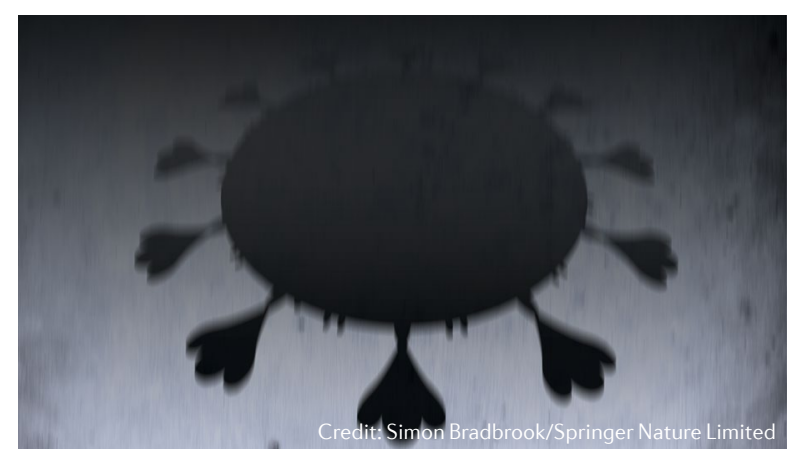

is known to be induced by inflammation. Moreover, fibroblasts were significantly increased, with a rapid expansion of $C T H R C 1^{+}$pathological fibroblasts, which were recently described as drivers of lung fibrosis.

The source of inflammation was further investigated in a mass-cytometry dataset (mRNA analysis is unsuitable for analysing post-translationally activated cytokines), which revealed that myeloid-derived IL- $1 \beta$ distinguishes COVID-19 from other viral or bacterial pneumonias and may contribute to the inflammation-induced impairment of alveolar epithelial regeneration.

The second study, by Delorey et al., presents single-cell atlases of 23 lung, 16 kidney, 16 liver and 19 heart autopsy samples from individuals who died of COVID-19. They also describe spatial atlases of 14 SARS-CoV-2-infected lungs, where host responses in regions with and without viral RNA were compared.

snRNA-seq of the lungs revealed substantial remodelling in the epithelial, immune and stromal compartments. This included a significant decrease in AT2 cells (possibly due to virus-induced cell death) and an increase in dendritic cells, macrophages, fibroblasts and lymphatic and vascular endothelial cells. Overall, they found widespread cell type-specific transcriptional changes, notably in CD16 ${ }^{+}$ monocytes, lymphatic endothelial, vascular endothelial, AT1 and AT2 cells. They also found an increase of cells with a 'pre-AT1 transitional cell state' gene signature, which is similar to the DATP gene signature, and amongst these identified a subset of putative $\mathrm{TP}^{2} 3^{+}$intrapulmonary basal-like progenitor cells. These cells had been described in mice infected with H1N1 influenza virus, where they act as an emergency cellular reserve for severely damaged alveoli. This indicates that regenerative strategies are initiated to re-establish alveolar epithelial cells lost to infection, but that a serial failure of epithelial progenitors to regenerate may eventually lead to lung failure.

The authors also analysed viral load and associated host responses at single-cell resolution. Viral RNA was mainly detected in mononuclear phagocytes and endothelial cells, but some mast cells, B cells and plasma cells were also SARS-CoV-2positive. The infected cells had distinct transcriptional programmes compared with uninfected cells.

In heart, kidney and liver, only very few viral RNA reads were detected. However, in the heart, both cell composition and gene programmes showed differences compared with healthy hearts, with a significant reduction in the proportion of cardiomyocytes and pericytes and an increase in vascular endothelial cells.

The authors also integrated their atlas with genome-wide association studies to identify common variants associated with COVID-19 severity and relate these to particular cell types or tissues. They found several gene loci that linked AT2 cells with severe COVID-19.

The authors of both publications emphasize that their studies are limited by their small sample size. Nevertheless, their work will contribute to a collection of studies with streamlined protocols and harmonized metadata that will provide a critical resource to dissect the pathophysiology of COVID-19.

Alexandra Flemming

ORIGINAL ARTICLES Melms, J. C. et al. A molecular single-cell lung atlas of lethal COVID-19. Nature https://doi.org/10.1038/s41586-021-03569-1 (2021)| Delorey, T. M. et al. COVID-19 tissue atlases reveal SARS-CoV-2 pathology and cellular targets. Nature https://doi.org/10.1038/s41586-021-03570-8 (2021) 